\title{
Gain dynamics of quantum dot devices for dual-state operation
}

Cite as: Appl. Phys. Lett. 104, 261108 (2014); https://doi.org/10.1063/1.4885383

Submitted: 05 March 2014 • Accepted: 15 June 2014 • Published Online: 01 July 2014

Y. Kaptan, H. Schmeckebier, B. Herzog, et al.

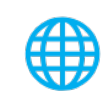

\section{ARTICLES YOU MAY BE INTERESTED IN}

Stability of quantum-dot excited-state laser emission under simultaneous ground-state perturbation

Applied Physics Letters 105, 191105 (2014); https://doi.org/10.1063/1.4901051

A dual-mode quantum dot laser operating in the excited state

Applied Physics Letters 99, 231110 (2011); https://doi.org/10.1063/1.3667193

Comparison of dynamic properties of ground- and excited-state emission in p-doped InAs/

GaAs quantum-dot lasers

Applied Physics Letters 104, 181101 (2014); https://doi.org/10.1063/1.4875238

母QBLOX

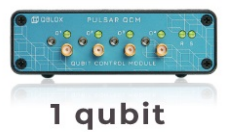

Shorten Setup Time Auto-Calibration More Qubits

Fully-integrated Quantum Control Stacks Ultrastable DC to $18.5 \mathrm{GHz}$ Synchronized $<<1$ ns Ultralow noise

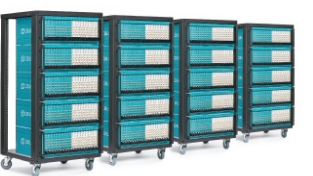

100s qubits

visit our website > 


\title{
Gain dynamics of quantum dot devices for dual-state operation
}

\author{
Y. Kaptan, ${ }^{1, a)}$ H. Schmeckebier, ${ }^{2}$ B. Herzog, ${ }^{1}$ D. Arsenijević, ${ }^{2}$ M. Kolarczik, ${ }^{1}$ V. Mikhelashvili, ${ }^{3}$ \\ N. Owschimikow, ${ }^{1}$ G. Eisenstein,, ${ }^{3}$ D. Bimberg, ${ }^{2}$ and U. Woggon ${ }^{1}$ \\ ${ }^{1}$ Institut für Optik und Atomare Physik, Technische Universität Berlin, Berlin, Germany \\ ${ }^{2}$ Institut für Festkörperphysik, Technische Universität Berlin, Berlin, Germany \\ ${ }^{3}$ Technion Institute of Technology, Faculty of Electrical Engineering, Haifa, Israel
}

(Received 5 March 2014; accepted 15 June 2014; published online 1 July 2014)

\begin{abstract}
Ground state gain dynamics of $\operatorname{In}(\mathrm{Ga})$ As-quantum dot excited state lasers are investigated via single-color ultrafast pump-probe spectroscopy below and above lasing threshold. Two-color pump-probe experiments are used to localize lasing and non-lasing quantum dots within the inhomogeneously broadened ground state. Single-color results yield similar gain recovery rates of the ground state for lasing and non-lasing quantum dots decreasing from 6 ps to 2 ps with increasing injection current. We find that ground state gain dynamics are influenced solely by the injection current and unaffected by laser operation of the excited state. This independence is promising for dual-state operation schemes in quantum dot based optoelectronic devices. (C) 2014
\end{abstract} AIP Publishing LLC. [http://dx.doi.org/10.1063/1.4885383]

Self-assembled InAs quantum dots (QDs) are widely used as an active medium in optoelectronic devices for telecommunication applications such as lasers and amplifiers. ${ }^{1,2}$ They provide ultrafast gain recovery, low threshold currents, and allow for multi wavelength amplification as well as pattern-free signal amplification with high bit rates and ultrafast nonlinear signal processing. ${ }^{3-6}$ For operation in the optical telecommunication windows, zero-dimensional (OD) InAs QDs are typically coupled to a two-dimensional (2D) carrier reservoir and embedded in a GaAs or InP matrix. Quantum dots emitting at $1.3 \mu \mathrm{m}$ support a confined ground state (GS) and one to several excited states (ES). ${ }^{7}$

QD-based devices typically operate on the QD GS, ${ }^{3-6}$ though ES operation and simultaneous or switchable dualstate operation has attracted considerable attention. ${ }^{8-16}$ While the ultrafast carrier dynamics of the GS single-state operating devices have been studied in a large number of works, ${ }^{17-27}$ to date no experiments on the interdependency of the ultrafast gain dynamics in dual-state or ES laser devices have been conducted. Especially interesting is the influence of different steady-state conditions and carrier lifetimes in the ES on the carrier relaxation into the GS. Unambiguous knowledge of the carrier relaxation pathways in these coupled 0D/2D systems is crucial for designing and engineering advanced QD lasers and semiconductor optical amplifiers with improved high-speed modulation performance, high-speed linear amplification, and high speed nonlinear signal processing, e.g., wavelength conversion. ${ }^{4}$

In this Letter, we experimentally investigate the influence of ES lasing on the QD GS gain recovery dynamics in devices which have been tailored to provide modified steady-state population conditions compared to standard GS-based devices. Furnished with a dichroic facet coating acting as antireflection coating in the GS, whereas highly reflecting in the $\mathrm{ES}$, these devices operate up to a certain injection current as optical amplifiers in the GS and

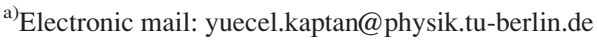

eventually reach laser threshold of the ES. We address the GS gain recovery in such a device using ultrafast pumpprobe (PP) experiments.

The investigated AlGaAs/GaAs based devices were grown by molecular beam epitaxy and contain 5 stacks of InGaAs/GaAs QDs in the active zone. Each dot layer is overgrown with an InGaAs quantum well (DWELL structure). ${ }^{28}$ The mesa consists of a shallow etched $1.33 \mathrm{~mm}$ long and $6 \mu \mathrm{m}$ wide ridge waveguide. The front facet (PP input into the device) is as cleaved, whereas the rear facet (PP output from the device) is coated.

The emission collected from a typical ES device for a series of injection currents is shown in Fig. 1. Size, shape, and composition distribution of the QDs lead to an inhomogeneous broadening of the luminescence. ${ }^{29,30}$ At low injection levels, we observe emission from the GS, centered at $1250 \mathrm{~nm}(0.992 \mathrm{eV})$ with a full width at half maximum

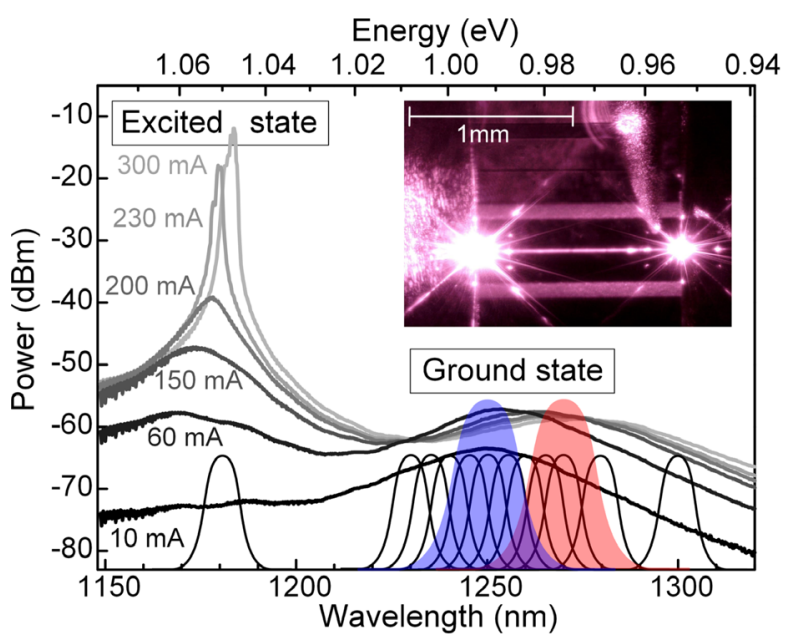

FIG. 1. Emission spectra of the QD device for a range of injection currents. GS and ES emission are clearly separated and the onset of ES lasing is at about $200 \mathrm{~mA}$. The energies of the single-color pump-probe experiments are illustrated by the colored Gaussians. For the two-color experiment, ES pump and GS probe ranges are represented by the bare Gaussian lines. Inset: Image of the lasing device visualized by a digital microscope. 
(FWHM) of $25 \mathrm{~nm}(20 \mathrm{meV})$. Increasing the injection current leads to an increase of the ES emission, centered at a wavelength of around $1180 \mathrm{~nm}(1.051 \mathrm{eV})$. Above a threshold current of about $200 \mathrm{~mA}$, a narrow laser line builds up on top of the ES emission.

Single- and two-color PP experiments in heterodyne configuration have been carried out to investigate the roomtemperature gain dynamics in the device at GS energies for injection currents below and above the ES lasing threshold, i.e., under fundamentally different steady-state situations. The pump and probe laser pulses were generated by a Toptica FemtoFiber Pro laser system, in which the emission line of a modelocked erbium-doped fiber laser is split and amplified independently in two nonlinear fibers to yield supercontinuum light at wavelengths between $900 \mathrm{~nm}$ and $2000 \mathrm{~nm}$. From these two supercontinua, suitable portions of Gaussian shape and about $15 \mathrm{~nm}$ and $10 \mathrm{~nm}$ spectral FWHM for the single-color and two-color experiments, respectively, are cut by an amplitude mask in the Fourier plane of a pulse shaper. A schematic of the setup is shown in Fig. 2. The pulses are chirp compensated and compressed to yield pump and probe pulses with a temporal duration of $250 \mathrm{fs}$ and $350 \mathrm{fs}$, respectively. The temporal resolution of the setup is limited only by this pulse width.

The pump pulse power in the experiment is about $500 \mu \mathrm{W}$, corresponding to $6.5 \mathrm{pJ}$ per pulse, at a repetition rate of $75.4 \mathrm{MHz}$. Pump and probe pulses are coupled into the device waveguide with an in-coupling efficiency of $<10 \%$, thus operating the device in the limit of the small signal amplification regime. Changes in transmission of the probe pulse are detected in a heterodyne balanced detection scheme $^{31}$ using a Nirvana 2017 auto-balanced photoreceiver and a fast $2 \mathrm{MHz}$ lock-in amplifier (Perkin Elmer DSP 7280). From the differential transmission data, we determine the pump-induced changes of the gain $\Delta \mathrm{G}=10 \log \left(T / T_{0}\right)$, with $T$ being the sample transmission at the probe wavelength in presence, and $T_{0}$ being the transmission in absence of the pump pulse.

As we aim to observe the change of gain dynamics of the GS due to ES lasing, we have to take into account the effects of the inhomogeneous broadening. As shown in Fig. 1, only a certain sub-ensemble of QDs is oscillating within the ES. It is, therefore, necessary to identify the

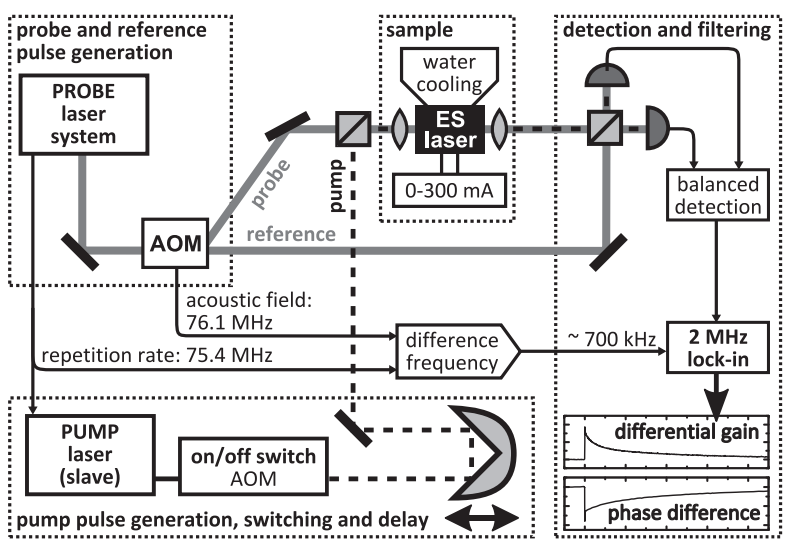

FIG. 2. Single- and two-color PP setup in heterodyne detection configuration with balanced detection. energy range in the GS, which corresponds to the QD subensemble lasing in its ES. To this end, we performed a series of preliminary two-color PP experiments without injection current. In these experiments, the ES is optically pumped, and the transmission signal in the GS for a range of energies is recorded. When pumping and probing the same QD subensemble, a direct intra-QD relaxation from ES to GS is expected to occur which manifests itself in an ultrafast change of the transmitted probe pulse. ${ }^{32,33}$ For different pumped and probed QD sub-ensembles, optically generated excitons need to pass through the commonly shared 2D carrier reservoir by up- and down-scattering processes, which are reflected by slower changes of the GS transmission. As mentioned before, the device is optically pumped at the ES laser line, while the probe pulse is tuned from $1230 \mathrm{~nm}$ $(1.008 \mathrm{eV})$ to $1310 \mathrm{~nm}(0.943 \mathrm{eV})$, covering the GS luminescence energies. An additional single-color PP measurement at $1180 \mathrm{~nm}$ is performed to resolve the expected ultrafast change in transmission when pumping and probing the same QD sub-ensemble. The energies and pulse widths for these measurements are schematically illustrated as Gaussian solid lines at the bottom of Fig. 1. In Fig. 3(a), exemplary normalized transmission traces measured at three representative probe energies of $1235 \mathrm{~nm}, 1255 \mathrm{~nm}$, and $1270 \mathrm{~nm}$ are shown. The expected pump-induced increase of the transmission signal is observed in all cases, and the rise-time of it depends on the probe wavelength. A plot of the measured $10 \%$ to $90 \%$ rise times, extracted from the pump-probe traces for all probe wavelengths is shown in Fig. 3(b). The measured rise-times smoothly decrease with increasing probe wavelength and ultimately reach 350 fs at wavelengths above $1260 \mathrm{~nm}$, which is the time resolution of the two-color PP experiment given by a convolution of pump and probe pulse durations represented by the shaded area in Figs. 3(a) and 3(b). For further increasing probe wavelengths, we again observe longer rise times. Since an ultrafast resolution limited $350 \mathrm{fs}$ response indicates an intra-dot process, while lateral dot-to-dot coupling is much slower, ${ }^{32,33}$ we are able to
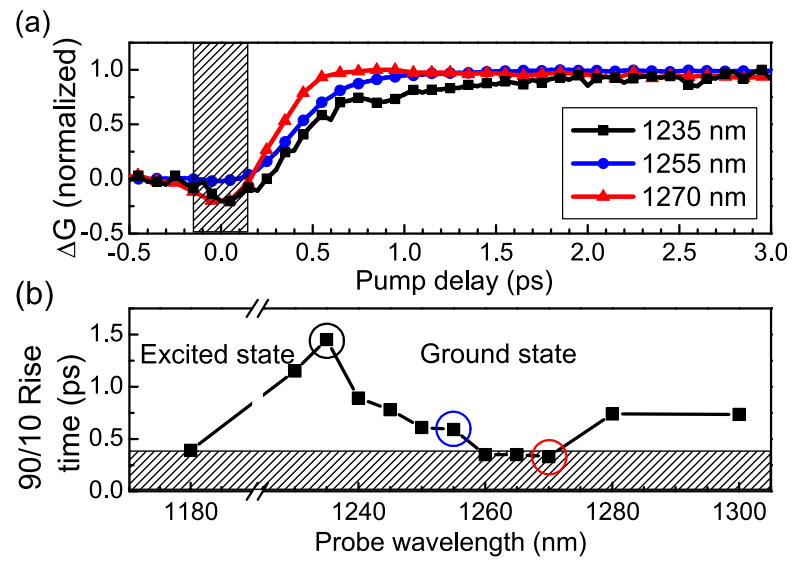

FIG. 3. (a) Normalized changes of the gain (respectively, probe transmission) at three representative probe wavelengths within the GS under optical excitation of the ES lasing QDs at $1180 \mathrm{~nm}$. The shaded area corresponds to the FWHM of the convolution of pump and probe pulse, i.e., the experimental time resolution of roughly $350 \mathrm{fs}$. (b) 90/10 rise-times of the pump-probe transients optically pumped at the ES laser line and probed in the GS between $1230 \mathrm{~nm}(1.008 \mathrm{eV})$ and $1300 \mathrm{~nm}(0.976 \mathrm{eV})$ as well as in the ES at $1180 \mathrm{~nm}$. The solid line is a guide to the eye. 
identify the ES lasing QD sub-ensemble with QDs emitting between 1260 and $1275 \mathrm{~nm}$.

In the following, single-color PP investigations of the GS gain recovery are performed at the wavelengths of $1255 \mathrm{~nm}(0.988 \mathrm{eV})$ and $1270 \mathrm{~nm}(0.976 \mathrm{eV})$. As confirmed above, the latter addresses the GS of QDs lasing in the ES, and the former is a control ensemble, for which the slower observed rise-time hints at the presence of QDs disconnected from the ES lasing. A representative set of gain traces measured at $1255 \mathrm{~nm}$ for a set of injection currents is displayed in Fig. 4(a). The traces depict the normalized pump-induced change of gain $\Delta \mathrm{G}$ and show a clearly non-monoexponential gain recovery, which can be partitioned into well separated time ranges. Three exponentials can be identified within the gain traces. First, within an ultrafast sub-picosecond time range coherent dynamics and coupled polarization between pump- and probe-pulses can be observed and analyzed within microscopic models, e.g., a Maxwell-Bloch equation approach. ${ }^{34,35}$ Carrier capture into the QD accounts for the technologically important fast gain recovery and manifests itself on an intermediate time scale of few ps. ${ }^{18,19}$ The slow exponential decay of some 100 ps is associated with slow carrier reservoir refilling via the externally applied current, injecting carriers into the barrier states. ${ }^{36}$ These carriers relax via phonon-mediated ${ }^{37,38}$ and Coulomb-mediated ${ }^{19,38,39}$ processes into the $2 \mathrm{D}$ carrier reservoir and slowly thermalize to a steady-state.

Due to the order of magnitude in time separation between the slow thermalization and the fast few-ps gain recovery, i.e., capture process into the QD states, the slow component is quasi a constant within the first tens of picoseconds. Thus, we are able to separate the slow component and fit the first $30 \mathrm{ps}$ of our gain traces with a two-exponential function and an additive offset $y_{0}$, with the fast time constant $\tau_{1}$ fixed to the experimental time resolution, corresponding
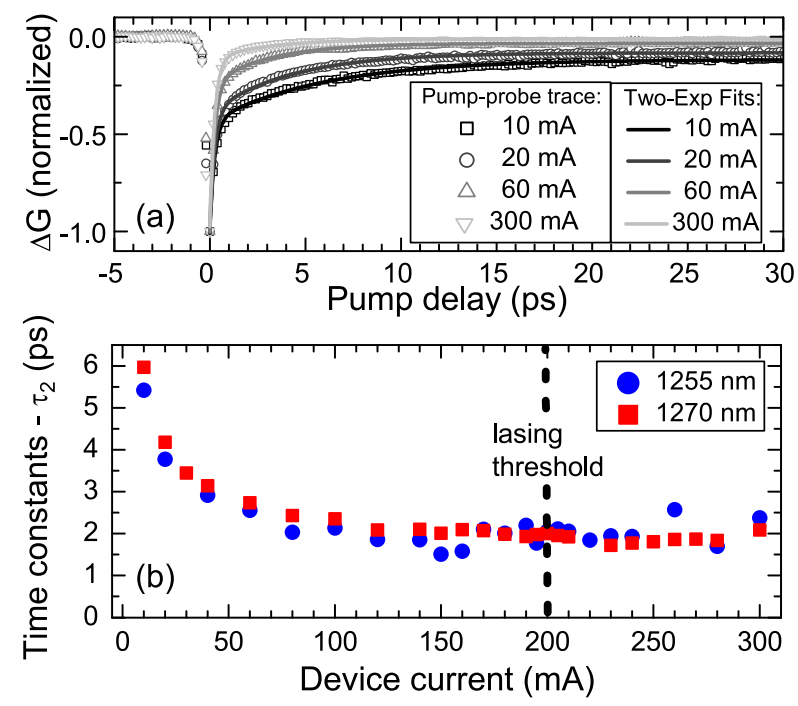

FIG. 4. (a) Pump-probe traces for a range of injection currents measured at $1255 \mathrm{~nm}(0.988 \mathrm{eV})$. Two-exponential fits of the data are shown as solid lines. (b) Time constants $\tau_{2}$ depending on injection current derived from fits of the single-color PP traces measured in the QD GS at $1255 \mathrm{~nm}(0.988)$ (blue dots) and $1270 \mathrm{~nm}(0.976 \mathrm{eV})$ (red squares). The error bars of the fits are on the order of the symbol sizes and left out. The vertical dashed line represents the lasing threshold. to the regime of coherent response of the system. All traces are well described by this two-exponential decay function given by $y=y_{0}+y_{1} e^{t / \tau_{1}}+y_{2} e^{t / \tau_{2}}$. Exemplary fits are shown for a representative set of curves as solid lines in Fig. 4(a).

The extracted second time constants $\tau_{2}$, describing the GS recovery dynamics, are shown in Fig. 4(b) in dependence of injection current as squared red and circular blue dots, representing the probe wavelengths of $1270 \mathrm{~nm}(0.976 \mathrm{eV})$ and $1255 \mathrm{~nm}(0.988 \mathrm{eV})$. We find that the GS recovery times are comparable to the ones published for similar GS-based devices. ${ }^{18,19,22}$ At low currents, we observe a 6 ps recovery time, which is decreasing to 2 ps for higher injection currents above $80 \mathrm{~mA}$ due to above mentioned Coulomb-mediated scattering processes. The dashed vertical line in Fig. 4(b) represents the laser threshold, cf. Fig. 1. Upon reaching and exceeding laser threshold, no difference in the gain recovery is observed for both the lasing $(1270 \mathrm{~nm})$ and non-lasing $(1255 \mathrm{~nm})$ QDs. The onset of ES lasing is accompanied by a sudden change of the light field oscillating in the QD device, which does not influence the GS recovery. Although the enhanced light field could potentially act as an additional optical carrier reservoir feeding the QD states after GS carrier depletion, ${ }^{40,41}$ no further decrease of the GS gain recovery rate is observed. We conclude that the recovery is rather governed by the present ES carrier population which reacts only weakly to crossing the laser threshold. ${ }^{15}$ From Fig. 4(b), it is clear that at high currents small changes in the ES population will not affect the GS gain recovery which reaches its optimum of 2 ps already at $80 \mathrm{~mA}$.

In conclusion, we experimentally investigated the process of GS gain recovery in a laser which oscillates only in the ES via ultrafast single- and two-color PP spectroscopy. The device was modified compared to the ones hitherto studied in the literature by introducing a dichroic mirror coating which leads to an enhanced feedback at the ES wavelength and thus to different steady-state conditions. We studied GS gain recovery at room temperature in sub-ensembles both strongly and weakly affected by this modification. We found recovery rates similar to the ones of standard GS-based quantum dot optical amplifiers, with the current dependence governed by Coulomb scattering and unaffected by crossing the ES laser threshold. The GS recovery rates become independent of injection current and ES lasing condition above a threshold current of about $80 \mathrm{~mA}$. This undisturbed performance of the ultrafast GS gain dynamics supports the capacity of quantum dot based devices for future dual-state operation schemes. A promising application is simultaneous laser and amplifier operation in different QD states by directly modulating the ES emission via current modulation without influencing gain recovery of the GS operating as semiconductor optical amplifier.

This work was supported by Sfb 787 from the German research foundation and the Alexander von Humboldt foundation.

${ }^{1}$ D. Bimberg and U. W. Pohl, Mater. Today 14, 388 (2011).

${ }^{2}$ J. P. Reithmaier, A. Somers, W. Kaiser, S. Deubert, F. Gerschütz, A. Forchel, O. Parillaud, M. Krakowski, R. Alizon, D. Hadass, A. Bilenca, H. Dery, V. Mikhelashvili, G. Eisenstein, M. Gioannini, I. Montrosset, T. W. Berg, M. van der Poel, J. Mørk, and B. Tromborg, Phys. Status Solidi B 243, 3981 (2006). 
${ }^{3}$ M. Matsuura, O. Raz, F. Gomez-Agis, N. Calabretta, and H. J. S. Dorren, Opt. Express 19, B551 (2011).

${ }^{4}$ H. Schmeckebier, C. Meuer, D. Arsenijević, G. Fiol, C. SchmidtLanghorst, C. Schubert, G. Eisenstein, and D. Bimberg, IEEE Photonics Technol. Lett. 24, 1163 (2012).

${ }^{5}$ G. Contestabile, Y. Yoshida, A. Maruta, and K. Kitayama, Opt. Express 20, 27902 (2012).

${ }^{6}$ A. Capua, S. O’Duill, V. Mikhelashvili, G. Eisenstein, J. P. Reithmaier, A. Somers, and A. Forchel, Opt. Express 16, 19072 (2008).

${ }^{7}$ F. Guffarth, R. Heitz, A. Schliwa, O. Stier, A. Kovsh, V. Ustinov, N. N. Ledentsov, and D. Bimberg, Phys. Status Solidi B 224, 61 (2001).

${ }^{8}$ H.-Y. Wang, H.-C. Cheng, S.-D. Lin, and C.-P. Lee, Appl. Phys. Lett. 90, 081112 (2007).

${ }^{9}$ M. A. Cataluna, D. I. Nikitichev, S. Mikroulis, H. Simos, C. Simos, C. Mesaritakis, D. Syvridis, I. Krestnikov, D. Livshits, and E. U. Rafailov, Opt. Express 18, 12832 (2010).

${ }^{10}$ F. Grillot, N. A. Naderi, J. B. Wright, R. Raghunathan, M. T. Crowley, and L. F. Lester, Appl. Phys. Lett. 99, 231110 (2011).

${ }^{11}$ M. Abusaa, J. Danckaert, E. A. Viktorov, and T. Erneux, Phys. Rev. A 87, 063827 (2013).

${ }^{12}$ A. Markus, M. Rossetti, V. Calligari, D. Chek-Al-Kar, J. X. Chen, A. Fiore, and R. Scollo, J. Appl. Phys. 100, 113104 (2006).

${ }^{13}$ L. W. Shi, Y. H. Chen, B. Xu, Z. C. Wang, and Z. G. Wang, Physica E 39, 203 (2007).

${ }^{14}$ K. Veselinov, F. Grillot, C. Cornet, J. Even, A. Bekiarski, M. Gioannini, and S. Loualiche, IEEE J. Quantum Electron. 43, 810 (2007).

${ }^{15}$ M. Gioannini, J. Appl. Phys. 111, 043108 (2012).

${ }^{16}$ V. V. Korenev, A. V. Savelyev, A. E. Zhukov, A. V. Omelchenko, and M. V. Maximov, Semiconductors 47, 1397 (2013).

${ }^{17}$ J. Gomis-Bresco, S. Dommers-Völkel, O. Schöps, Y. Kaptan, O. Dyatlova, D. Bimberg, and U. Woggon, Appl. Phys. Lett. 97, 251106 (2010).

${ }^{18}$ S. Dommers, V. V. Temnov, U. Woggon, J. Gomis, J. Martinez-Pastor, M. Laemmlin, and D. Bimberg, Appl. Phys. Lett. 90, 033508 (2007).

${ }^{19}$ J. Gomis-Bresco, S. Dommers, V. V. Temnov, U. Woggon, J. MartinezPastor, M. Laemmlin, and D. Bimberg, IEEE J. Quantum Electron. 45, 1121 (2009).

${ }^{20}$ T. Piwonski, I. O’Driscoll, J. Houlihan, G. Huyet, R. J. Manning, and A. V. Uskov, Appl. Phys. Lett. 90, 122108 (2007).

${ }^{21}$ T. Erneux, E. A. Viktorov, P. Mandel, T. Piwonski, G. Huyet, and J. Houlihan, Appl. Phys. Lett. 94, 113501 (2009).

${ }^{22}$ J. Kim, M. Laemmlin, C. Meuer, D. Bimberg, and G. Eisenstein, IEEE J. Quantum Electron. 45, 240 (2009).

${ }^{23}$ D. Hadass, A. Bilenca, R. Alizon, H. Dery, V. Mikhelashvili, G. Eisenstein, R. Schwertberger, A. Somers, J. P. Reithmaier, A. Forchel,
M. Calligaro, S. Bansropun, and M. Krakowski, IEEE J. Sel. Top. Quantum Electron. 11, 1015 (2005).

${ }^{24}$ P. Borri, S. Schneider, W. Langbein, and D. Bimberg, J. Opt. A: Pure Appl. Opt. 8, S33 (2006).

${ }^{25}$ A. J. Zilkie, J. Meier, M. Mojahedi, P. J. Poole, P. Barrios, D. Poitras, T. J. Rotter, C. Yang, A. Stintz, K. J. Malloy, P. W. E. Smith, and J. S. Aitchison, IEEE J. Quantum Electron. 43, 982 (2007).

${ }^{26}$ M. van der Poel and J. M. Hvam, J. Mater. Sci. Mater. Electron. 18, 51 (2007).

${ }^{27}$ R. P. Prasankumar, W. W. Chow, J. Urayama, R. S. Attaluri, R. V. Shenoi, S. Krishna, and A. J. Taylor, Appl. Phys. Lett. 96, 031110 (2010).

${ }^{28}$ A. R. Kovsh, N. A. Maleev, A. E. Zhukov, S. S. Mikhrin, A. P. Vasil'ev, E. A. Semenova, Y. M. Shernyakov, M. V. Maximov, D. A. Livshits, V. M. Ustinov, N. N. Ledentsov, D. Bimberg, and Z. I. Alferov, J. Cryst. Growth 251, 729 (2003).

${ }^{29}$ N. Kirstaedter, O. G. Schmidt, N. N. Ledentsov, D. Bimberg, V. M. Ustinov, A. Y. Egorov, A. E. Zhukov, M. V. Maximov, P. S. Kop'ev, and Z. I. Alferov, Appl. Phys. Lett. 69, 1226 (1996).

${ }^{30}$ J. Shumway, A. J. Williamson, A. Zunger, A. Passaseo, M. DeGiorgi, R. Cingolani, M. Catalano, and P. Crozier, Phys. Rev. B 64, 125302 (2001).

${ }^{31}$ P. Borri, W. Langbein, J. Mørk, and J. M. Hvam, Opt. Commun. 169, 317 (1999).

${ }^{32}$ K. L. Silverman, R. P. Mirin, and S. T. Cundiff, Phys. Rev. B 70, 205310 (2004).

${ }^{33}$ I. O'Driscoll, T. Piwonski, C.-F. Schleussner, J. Houlihan, G. Huyet, and R. J. Manning, Appl. Phys. Lett. 91, 071111 (2007).

${ }^{34}$ M. Wegert, N. Majer, K. Lüdge, S. Dommers-Völkel, J. Gomis-Bresco, A. Knorr, U. Woggon, and E. Schöll, Semicond. Sci. Technol. 26, 014008 (2011).

${ }^{35}$ E. Malic, M. Richter, G. Hartmann, J. Gomis-Bresco, U. Woggon, and A. Knorr, New J. Phys. 12, 063012 (2010).

${ }^{36}$ N. Majer, K. Lüdge, J. Gomis-Bresco, S. Dommers-Völkel, U. Woggon, and E. Schöll, Appl. Phys. Lett. 99, 131102 (2011).

${ }^{37}$ B. Ohnesorge, M. Albrecht, J. Oshinowo, A. Forchel, and Y. Arakawa, Phys. Rev. B 54, 11532 (1996).

${ }^{38}$ A. Capua, O. Karni, and G. Eisenstein, IEEE J. Sel. Top. Quantum Electron. 19, 1900410 (2013).

${ }^{39}$ J. Gomis-Bresco, S. Dommers, V. V. Temnov, U. Woggon, M. Laemmlin, D. Bimberg, E. Malic, M. Richter, E. Schöll, and A. Knorr, Phys. Rev. Lett. 101, 256803 (2008).

${ }^{40}$ J. Kim, J. Opt. 15, 105402 (2013).

${ }^{41}$ H. Baghban, R. Maram Q, R. Oliaee, R. Yadipour, and A. Rostami, J. Opt. 13, 035406 (2011). 